UMN-TH-1805/99

TPI-MINN-99/31

hep-ph/9906331

June 1999

\title{
Assisted Chaotic Inflation in Higher Dimensional Theories
}

\author{
Panagiota Kanti and Keith A. Olive \\ Theoretical Physics Institute, School of Physics and Astronomy, \\ University of Minnesota, Minneapolis, MN 55455, USA
}

\begin{abstract}
We address the problem of the large initial field values in chaotic inflation and propose a remedy in the framework of the so-called assisted inflation. We demonstrate that a 4-dimensional theory of multiple, scalar fields with initial field values considerably below the Planck scale, can give rise to inflation even though none of the individual scalar fields are capable of driving inflation. The problems arising from the presence of possible non-renormalizable interactions is therefore removed. As a concrete example of a theory with multiple scalar fields, we consider a $(4+d)$-dimensional field theory of a single, noninteracting massive scalar field whose KK modes play the role of the assisted sector. For the KK modes to assist inflation, the extra dimensions must have a size larger than the inverse (4D) Planck scale.
\end{abstract}

Emails: yiota@physics.umn.edu, olive@mnhep.hep.umn.edu 


\section{Introduction}

Barring an alternative, inflation is an essential component of the standard big bang cosmological model. Because there is no firm model of inflation, particularly from a particle physics point of view, the literature is abound with models of inflation [1]. Without a doubt, the simplest of these models is the chaotic inflationary scenario [2]. This model is based on a single scalar field and can be formulated either as a theory of a massless scalar with a quartic self-coupling $\lambda$ or a non-interacting massive scalar with mass $m$. In either variety, the magnitude of the density perturbations produced by quantum fluctuations of the scalar field during its evolution allows one to fix either the mass or quartic coupling, $m \sim 10^{-5} M_{P}$ (where $M_{P}$ is the Planck mass) or $\lambda \sim 10^{-12}$.

The initial conditions for chaotic inflation do not rely on an initial thermal state. Rather, chaotic inflation is based on the simple assumption that the initial vacuum energy density is of the Planck scale, that is $V(\phi) \simeq M_{P}^{4}$. Inflation occurs for $\phi \gtrsim M_{P}$ and its duration will be long enough to solve the cosmological problems for $\phi>$ a few $M_{P}$. Of course, the condition on the vacuum density implies $\phi \gg M_{P}$, and there will in general be significantly more inflation than the necessary minimum. It is precisely this condition on the initial values of the inflaton, $\phi_{0}$, that can in principle be troublesome. For example, in order to assume $\phi_{0} \gg M_{P}$ and use only the quadratic or quartic potential terms, one is implicitly assuming that all non-renormalizable terms (whether at the tree level or arising at one or more loops) are absent or severely suppressed[1]3].

Recently, it was shown [5] that a system of several scalar fields each with an exponential potential could drive power-law inflation with a net power greater than the one achieved by a single field. Indeed, it is possible to show that the inflation driven by such a system of multiple fields is sufficient to solve the cosmological problems, even if each of the individual fields $\phi_{i}$ alone is not capable of doing so. This idea was extended to quartic and quadratic potentials in [6], where it was shown that the fine-tuning of the quartic coupling $\lambda \sim$ $10^{-12}$ could be relaxed in the framework of a 4-dimensional theory of multiple scalar fields. However, it was also shown that these fields must not be cross coupled, otherwise, their multiplicity actually impedes inflation [6, 7]. The question of density perturbations (both adiabatic and isocurvature) in assisted inflation was discussed in [8].

In [6], we also argued that the source of a large number of nearly identical scalar fields

\footnotetext{
${ }^{1} \mathrm{~A}$ more complicated picture involving two or more scalar fields can relieve the problem of extremely large initial field values 輎.
} 
could be the compactification of a (relatively) large extra dimension. In this case, although the tower of KK states are heavily cross coupled, an attractor solution to the equations of motion was found such that the fine-tuning of the quartic coupling could be relaxed. Here, we will explore the effects of the large number of scalar fields that arise from the compactification of a theory containing a $(4+d)$-dimensional non-interacting massive field In particular, we will focus on a remedy of another potential problem concerning chaotic inflation, that of the large initial conditions on the value of the inflaton field. We will show that the system of equations of motion will admit to inflationary solutions for which the initial field values of all the scalar fields is significantly below the Planck scale, and as a result are not affected by any possible non-renormalizable interactions.

\section{Assisted inflation and initial conditions}

Although we will be mainly interested in an $m^{2} \phi^{2}$ chaotic inflationary model, with the aim of relaxing the initial conditions on the inflaton $\phi$, we begin by considering a general $m^{2} \phi^{2}+\lambda \phi^{4}$ potential. In addition, let us consider a theory with multiple, self-interacting scalar fields with a Lagrangian given by the expression

$$
-\mathcal{L}=\sum_{i=1}^{N}\left\{\frac{1}{2} \partial_{\mu} \phi_{i} \partial^{\mu} \phi_{i}+\frac{1}{2} m^{2} \phi_{i}^{2}+\frac{\lambda}{4 !} \phi_{i}^{4}\right\},
$$

with each field satisfying the equation of motion

$$
\ddot{\phi}_{i}+3 H \dot{\phi}_{i}=-\frac{d V_{i}}{d \phi_{i}}=-m^{2} \phi_{i}-\frac{\lambda}{3 !} \phi_{i}^{3}
$$

The Hubble parameter is given by

$$
H^{2}=\frac{8 \pi G}{3} \sum_{i=1}^{N}\left\{\frac{1}{2} \dot{\phi}_{i}^{2}+\frac{1}{2} m^{2} \phi_{i}^{2}+\frac{\lambda}{4 !} \phi_{i}^{4}\right\} .
$$

The equations of motion of any two scalar fields $\phi_{i}$ and $\phi_{j}$ are solved by a simple scaling solution and the unique late-time attractor of the system is given by $\phi_{i}=\phi_{j}$. In terms of this solution, which is valid at all times, the Lagrangian can be written as [6]

$$
-\mathcal{L}=N\left\{\frac{1}{2} \dot{\phi}_{1}{ }^{2}+\frac{m^{2}}{2} \phi_{1}^{2}+\frac{\lambda}{4 !} \phi_{1}^{4}\right\}=\frac{1}{2} \dot{\tilde{\phi}}^{2}+\frac{\tilde{m}^{2}}{2} \tilde{\phi}^{2}+\frac{\tilde{\lambda}}{4 !} \tilde{\phi}^{4}
$$

\footnotetext{
${ }^{2}$ For recent work concerning inflation and large extra dimensions, see [9]. In [10], KK-modes were used in constructing inflationary models in the context of higher derivative gravity models.
} 
where

$$
\tilde{\phi}=\sqrt{N} \phi_{1}, \quad \tilde{m}^{2}=m^{2}, \quad \tilde{\lambda}=\frac{\lambda}{N} .
$$

Thus, the above field redefinition allows us to rewrite the Lagrangian in terms of a single scalar field with a canonical kinetic term. Note that the mass of the renormalized scalar field, $\tilde{\phi}$, is unchanged though its quartic coupling is reduced by a factor $N$. This allows one to relax the fine-tuning of the quartic coupling and, at the same time, remain consistent with the energy density fluctuations observed in the micro-wave background.

As we noted in the introduction, if $\tilde{\phi}$ satisfies the initial condition, $\tilde{\phi}_{0} \gtrsim 3(4.5) M_{P}$, the theory described by either the quadratic (quartic) terms in (4) is well known to lead to a period of inflationary expansion of the universe with time-scale

$$
H \tau=\frac{2 \pi \tilde{\phi}_{0}^{2}}{M_{P}^{2}}\left(\frac{\pi \tilde{\phi}_{0}^{2}}{M_{P}^{2}}\right)>65 .
$$

However, the field $\tilde{\phi}$ that plays the role of the inflaton is only an effective field constructed

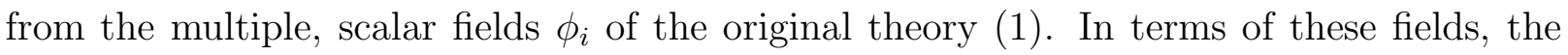
initial condition on $\tilde{\phi}$ translates to

$$
\left(\phi_{i}\right)_{0} \gtrsim \frac{3(4.5) M_{P}}{\sqrt{N}}
$$

which means that, for a large number of multiple fields, the initial value of each field $\phi_{i}$ can be considerably smaller than $M_{P}$, thus relieving the concern regarding the role (or magnitude) of possible non-renormalizable contributions to the Lagrangian. As a result, the multiplicity of a theory with respect to the number of scalar fields that it contains may resolve the problem of large initial conditions necessary for chaotic inflation as long as the masses of these fields are equal.

\section{$3 \quad$ Kaluza-Klein reduction of a $\hat{m}^{2} \hat{\phi}^{2}$ theory}

As a possible source of a field theory with multiple scalar fields, we consider a $(4+d)$ dimensional gravitational theory of the form

$$
S_{4+d}=-\int d^{4+d} x \sqrt{G_{4+d}}\left\{\frac{M^{2+d}}{16 \pi} R_{4+d}+\frac{1}{2} G_{4+d}^{A B} \partial_{A} \hat{\phi} \partial_{B} \hat{\phi}+\frac{1}{2} \hat{m}^{2} \hat{\phi}^{2}+\frac{\hat{\lambda}}{4 !} \frac{\hat{\phi}^{4}}{M^{d}}\right\}
$$

where $A, B=\left\{t, x_{1}, x_{2}, x_{3}, z_{1}, z_{2}, \ldots, z_{d}\right\}$ and $M$ is the $(4+d)$-dimensional Planck mass. The Kaluza-Klein reduction of the above theory will lead to the appearance of additional scalar 
fields, the Kaluza-Klein modes, in the framework of the 4-dimensional effective theory. If we assume that the extra $d$ dimensions are compactified over an internal manifold with the size of every compact dimension parametrized by $L$, we can Fourier expand the scalar field $\hat{\phi}$ along the compact dimensions in the following way

$$
\hat{\phi}(x, z)=\hat{\phi}_{0}(x)+\hat{\phi}_{z}(x, z)=\hat{\phi}_{0}(x)+\frac{1}{\sqrt{2}} \sum_{\vec{n}}\left(\hat{\phi}_{\vec{n}}(x) e^{i \frac{\pi \vec{n}}{L} \vec{z}}+\hat{\phi}_{\vec{n}}(x) e^{-i \frac{\pi \vec{n}}{L} \vec{z}}\right)
$$

where $\vec{n}=\left\{n_{1}, n_{2}, \ldots, n_{d}\right\}$ and $\hat{\phi}_{0}$ is the zero-mode that depends only on non-compact coordinates. Note that there is a maximum value of every $n_{i}$ which is defined by $n_{i} \leq$ $N \simeq L M$, corresponding to momenta $p_{i}=M$. In [6], we assumed that the mass $\hat{m}$ was zero and that the potential of the 5 -dimensional field $\hat{\phi}$ was characterized by a quartic selfinteraction term. After compactification, the system of the resulting Kaluza-Klein states is heavily cross coupled. Nevertheless, we were able to solve for an attractor solution of the system in terms of which the theory of multiple, cross coupled scalar fields was mapped into a theory of a single, self-interacting, field (through a quartic potential). The corresponding coupling constant was given by the expression

$$
\lambda=\hat{\lambda}\left(\frac{M_{5}}{M_{P}}\right)^{2}
$$

which relates the 4-dimensional, effective quartic coupling constant and Planck mass with the corresponding quantities of the original 5-dimensional theory (the analysis of [6] can be easily extended to an arbitrary number of extra dimensions and the above formula remains unchanged). Bearing in mind that the smallest possible value of the 5-dimensional Planck mass is $M_{5} \sim 10^{-6} M_{P}$ for inflation to occur [6], it is easy to see that a suppression factor of $10^{-12}$ comes directly from the ratio $\left(M_{5} / M_{P}\right)^{2}$ implying that $\hat{\lambda}$ can be of $\mathcal{O}(1)$. Just as one can consider the 4-dimensional gravitational scale characterized by $M_{P}$, to be an effective scale related to a fundamental, higher-dimensional energy scale and the size of the relatively large, extra dimensions by

$$
M_{P}^{2}=L^{d} M^{2+d}
$$

the 4-dimensional quartic coupling constant $\lambda$ can be considered as an effective coupling arising from the compactification of a higher-dimensional, fundamental theory.

A simple set of (non-cross-coupled) scalar fields with quartic self-couplings, as described in the previous section, allows one to scale-down of the initial field values while still providing chaotic inflation so long as eq. (7) is satisfied. The fact that the system of the multiple 
Kaluza-Klein states in [6] were non-trivially coupled, precludes such a solution. Instead of a solution of the form given in eq. (5) defining the inflaton, we found that for large $N$, $\tilde{\phi} \simeq \sqrt{1+\frac{1}{N}} \phi_{0}$, where $\phi_{0}$ is the zero KK mode of the theory 3 . Here, we limit our discussion to a non-interacting massive scalar field in $(4+\mathrm{d})$ dimensions and set $\hat{\lambda}=0$. The resulting 4-dimensional, effective action is then

$$
\begin{gathered}
S_{\text {eff }}=-\int d^{4} x \sqrt{g}\left\{\frac{M_{P}}{16 \pi} R+\frac{1}{2} \partial_{\mu} \phi_{0} \partial^{\mu} \phi_{0}+\frac{1}{2} \sum_{\vec{n}=1}^{N} \partial_{\mu} \phi_{\vec{n}} \partial^{\mu} \phi_{\vec{n}}+\right. \\
\left.\frac{1}{2} m^{2} \phi_{0}^{2}+\frac{1}{2} \sum_{\vec{n}=1}^{N}\left(m^{2}+m_{\vec{n}}^{2}\right) \phi_{\vec{n}}^{2}\right\},
\end{gathered}
$$

where we have made use of the following relations

$$
\phi=L^{d / 2} \hat{\phi}, \quad m^{2}=\hat{m}^{2}
$$

between the $(4+d)$-dimensional and the 4-dimensional quantities, of the fundamental relation (11) and of the definition

$$
m_{\vec{n}}^{2}=\frac{\vec{n}^{2} \pi^{2}}{L^{2}}
$$

for the Kaluza-Klein masses. In addition, the compactification of the extra dimensions gives rise to a dilaton-like field $\gamma$ and a gauge field $A_{\mu}$ defined as $G_{i i}=e^{\gamma}$, for $i=5, \ldots, d$, and $G_{\mu d}=e^{\gamma} A_{\mu}$, respectively. We will, however, ignore the gauge field and assume that the dilaton-like field remains fixed [11]. Note that, unlike the $\hat{\lambda} \hat{\phi}^{4}$ case [6], the resulting 4dimensional system we consider here, is still a simple set of non-interacting, massive scalar fields. We will show that this system leads to the relaxation of the large initial conditions according to the recipe of section 2 .

The zero-mode field $\phi_{0}$ and the higher Kaluza-Klein excitations $\phi_{\vec{n}}$ satisfy the equations of motion

$$
\begin{aligned}
\ddot{\phi}_{0}+3 H \dot{\phi}_{0} & =-m^{2} \phi_{0}, \\
\ddot{\phi}_{\vec{n}}+3 H \dot{\phi}_{\vec{n}} & =-\left(m^{2}+m_{\vec{n}}^{2}\right) \phi_{\vec{n}},
\end{aligned}
$$

respectively. Note that, although the bare mass $m^{2}$ has remained unchanged after the compactification of the extra dimensions, the appearance of a Kaluza-Klein mass for the

\footnotetext{
${ }^{3}$ There is a solution for which $\tilde{\phi} \simeq \sqrt{1+N} \phi_{0}$, but in this case the fine-tuning problem of the quartic coupling is not resolved and $\lambda=\hat{\lambda}$.
} 
higher modes of the theory leads, in principle, to the existence of a finite tower of scalar fields with different masses.

In principle, the multiple scalar fields of the 4-dimensional effective theory (12) can have arbitrary initial values. If we assume that all of the KK fields have initial values larger than $3 M_{P}$, each field of the theory can drive inflation independently of the presence of all other fields. As an illuminating example, we consider the case of two scalar fields, $\phi_{1}$ and $\phi_{2}$, with masses $m_{1}$ and $m_{2} \gg m_{1}$, respectively, that satisfy the following equations of motion

$$
\begin{aligned}
& \ddot{\phi}_{1}+3 H \dot{\phi}_{1}=-m_{1}^{2} \phi_{1}, \\
& \ddot{\phi}_{2}+3 H \dot{\phi}_{2}=-m_{2}^{2} \phi_{2} .
\end{aligned}
$$

Since both of the scalar fields have initial values larger than $3 M_{P}$, the slow roll-over conditions are satisfied for each of them and the energy density of the system is dominated by their mass terms. If we ignore the second derivatives of the fields with respect to time and rearrange their equations of motion, the following scaling solution is found

$$
\frac{\dot{\phi}_{1}}{\dot{\phi}_{2}}=\frac{m_{1}^{2}}{m_{2}^{2}} \frac{\phi_{1}}{\phi_{2}} \Rightarrow \phi_{1}(t) \sim \phi_{2}(t)^{m_{1}^{2} / m_{2}^{2}} .
$$

From the above relation, and since $m_{1}^{2} / m_{2}^{2} \ll \mathcal{O}(1)$, we may easily conclude that the light field $\phi_{1}$ evolves much slower towards its minimum value than the heavy field $\phi_{2}$.

In the present case, where a tower of Kaluza-Klein states with masses ranging from $m^{2}$ to $m^{2}+M^{2}$ satisfy the slow roll-over conditions, the Universe may undergo one or more periods of inflation depending on the initial field values, $\phi_{i}(0)$. Because of the scaling (19), the final period of inflation is typically driven by the lightest of the scalar fields under consideration (see e.g. [12]). For certain initial field configurations, however, quantum fluctuations may drive heavier scalar fields back to configurations which will subsequently inflate [13]. We will return to this issue below.

We are most interested in the case when all of the scalar fields have initial values less than $3 M_{P}$. In this case, the slow roll-over conditions can not be satisfied for any of the individual fields and, as a result, none of them can drive inflation alone. The equations of motion of the Kaluza-Klein fields (15)-(16) preclude a scaling solution that holds between different fields, at all times, as long as the masses of the fields are different. Moreover, the unique late-time attractor of the system is the trivial one, $\phi_{i}=\phi_{j}=0$. Thus, each field evolves towards its minimum independently of all the others, whose presence become manifest only through their contribution to the Hubble parameter. The duration of the 
roll-over phase for each field is proportional to its initial value and inversely proportional to its mass.

However, there is a subset of Kaluza-Klein fields which can be considered to have approximately the same mass and whose evolution, although not different from the picture described above, may give rise to an inflationary period for the universe. For the lightest of the Kaluza-Klein fields, the mass term $m_{\vec{n}}^{2}$ generated by the compactification of the extra dimensions can be considered negligible compared to the bare mass $m^{2}$. As a result, these fields can be grouped together, as in the model described in section 2, and form an effective inflaton field, $\tilde{\phi}$. For a large number of fields participating in the construction of $\tilde{\phi}$, the initial value of the inflaton may exceed the threshold of $3 M_{P}$ producing inflation while the values of the constituent fields remain well below the aforementioned threshold.

If we generalize the chaotic initial condition, $V(\phi) \sim M_{P}^{4}$, to the higher dimensional theory, we may write that $\hat{V}(\hat{\phi}) \sim M^{4+d}$. Then, in terms of 4-dimensional quantities, we obtain

$$
V(\tilde{\phi})=L^{d} \hat{V}(\hat{\phi}) \sim M_{P}^{2} M^{2} \sim m^{2} \tilde{\phi}^{2}
$$

If $\tilde{\phi}$ is to drive inflation, we must assume that $\tilde{\phi} \geq M_{P}$ and $m \sim 10^{-5} M_{P}$. Then, eq. (20) provides the constraint $M \geq 10^{-5} M_{P}$ on the higher dimensional Planck mass. Furthermore, the value of $M$ determines the number of Kaluza-Klein fields that can be considered to have approximately the same mass. Comparing the terms $m^{2}$ and $m_{\vec{n}}^{2}$, it is easy to conclude that the number of states that can be considered to have the same mass $m$ must satisfy the constraint

$$
\vec{n}_{m}^{2}<\frac{m^{2}}{M^{2}}\left(\frac{M_{P}}{M}\right)^{4 / d} \simeq \frac{m^{2}}{M^{2}}\left(N_{d}\right)^{2 / d},
$$

where $N_{d} \equiv(N+1)^{d} \simeq N^{d}=M_{P}^{2} / M^{2}$ is the total number of Kaluza-Klein states in the theory. For $d=1$, the actual number $N_{m}$ of Kaluza-Klein states that have approximately the same mass and, thus, participate in the construction of the inflaton field, is given by $N_{m}=\sqrt{\vec{n}_{m}^{2}}$. For the smallest possible value of the higher dimensional Planck mass, $M \sim 10^{-5} M_{P}, N_{m} \simeq N_{d} \sim 10^{10}$ while for $M \sim 10^{-3} M_{P}, N_{m} \simeq 10^{-2} N_{d} \sim 10^{4}$. In the former case, all of the KK fields participate in assisting inflation and the result is impressive. From eq. (5), the initial values of the constituent fields $\phi_{\vec{n}}$ can be as much as $10^{5}$ times smaller than $M_{P}$ (i.e. of order the 5-dimensional Planck scale) and still be able to produce inflation. When the value of $M$ is increased to $10^{-3} M_{P}$, only $1 \%$ of the KK fields participate. However, even in the latter case, the relaxation of the large initial conditions is still significant since the initial values of the constituent fields can, now, be approximately 
100 times smaller than $M_{P}$.

For more than one extra dimensions, the right-hand-side of eq. (21) becomes considerably smaller when compared to the case $d=1$. However, there is a degeneracy of states for $d>1$ that results in the existence, by construction, of multiple states with the same mass and, to a large extent, compensates the reduction in the rhs of eq. (21). The number of states in general will be $N_{m} \propto\left(\vec{n}_{m}^{2}\right)^{d / 2}$. Therefore the number of states is $N_{m} \propto(m / M)^{d} N_{d}$, and for $M \sim m$, the number of states is the same in any dimension. As a result, the construction of the inflaton field and the relaxation of its large initial conditions is still feasible, for an arbitrary number of extra dimensions, although less efficient as $d$ increases if $M>m$.

Let us note, that, during the inflationary period driven by the inflaton light field $\tilde{\phi}$, scalar field quantum fluctuations [13] are generated which may affect the evolution of the heavy KK fields which would otherwise evolve towards their minimum without producing inflation. These quantum fluctuations may increase the initial values of the heavy fields above the threshold of $3 M_{P}$ enabling them to drive inflation. If we assume that $m_{i}^{2} \ll H^{2}$, the quantum fluctuations grow as $H^{3} t / 4 \pi^{2}$ to a limiting value of

$$
\phi_{i}^{2} \simeq \frac{2}{3} \frac{m^{4} \tilde{\phi}_{0}^{4}}{M_{P}^{4}\left(m^{2}+m_{i}^{2}\right)},
$$

where $i$ denotes all the heavy Kaluza-Klein fields with masses equal to or larger than $m^{2}$. If the slow roll-over conditions are satisfied for any of the heavy fields, they could drive a new period of inflation. This is potentially problematic since they would produce an unacceptably large size for the density perturbations. As long as we restrict the initial value of the inflaton by

$$
\phi_{i}^{2} \lesssim 9 M_{P}^{2} \quad \text { or } \quad \tilde{\phi}_{0} \lesssim 3^{3 / 4} M_{P}\left(M_{P} / m\right)^{1 / 2} \simeq 720 M_{P}
$$

late periods of inflation driven by the heavy fields would not occur. These conditions are easily satisfied if we assume that all of the light fields have initial values suitably below the Planck scale.

\section{Conclusions}

Although the chaotic inflationary scenario is undoubtly the simplest model for inflation, it relies on the assumption that the initial values of the inflaton are large (compared to $M_{P}$ ) 
in order to obtain a period of rapid expansion long enough to solve existing cosmological problems. Moreover, the fundamental parameters of such a theory, being either the mass $m$ of a non-interacting scalar field or the quartic self-coupling $\lambda$ of the inflaton, must be extremely small in order to ensure that the density perturbations produced during inflation have the right order of magnitude. In a previous article [6], we demonstrated that a 4dimensional theory of multiple scalar fields can lead to chaotic inflation where the coupling constant $\lambda$ is small due to the multiplicity of the theory with respect to the scalar fields. As a concrete example of such a theory, we considered a 5-dimensional field theory where the Kaluza-Klein modes of the 5-dimensional scalar field played the role of the multiple fields. However, no remedy for the problem of large initial conditions was found in the context of the above theory due to the cross-coupling terms between the KK modes.

Here, we focused on the problem of large initial conditions. We demonstrated that a 4-dimensional field theory of multiple, self-interacting scalar fields can both resolve the finetuning problem of the quartic coupling and relax the initial conditions on the value of the inflaton field. The method is based on the construction of an effective field - the inflaton - from the original, multiple fields of the theory [5]. In this case, the inflaton has the same mass as the constituent fields but with an enhanced field value and a considerably weaker self-interaction. The method becomes more effective as the number of fields that participate in the construction of the inflaton becomes larger. As a result, for a large number of fields present in the theory, the quartic coupling constant assumes naturally a small value in agreement to the COBE data. Moreover, even for initial values of the constituent fields many orders of magnitude smaller than $M_{P}$, the value of the inflaton field can exceed the threshold of few $M_{P}$ and produce inflation.

As a concrete example of a theory with multiple scalar fields, we considered a $(4+d)$ dimensional field theory of a single, massive, non-interacting scalar field. Upon performing a Kaluza-Klein compactification of the extra dimensions, a 4-dimensional, effective theory of multiple, massive, non-interacting fields emerged. The appearance of a Kaluza-Klein mass for each one of the higher modes of the theory led to the existence of a finite tower of scalar fields with different masses. However, depending on the value of the higherdimensional Planck mass $M$, many of these fields can be considered to have approximately the same mass and, thus, give rise to an effective field that plays the role of the inflaton. For $M \sim 10^{-5} M_{P}$ or $M \sim 10^{-3} M_{P}$, the initial values of the original fields of the theory can be $10^{-5} M_{P}$ or $10^{-2} M_{P}$ respectively, and still enable the inflaton field to drive inflation. This removes any potential problem concerning the presence of non-renormalizable interactions 
in the theory.

Acknowledgments This work was supported in part by DOE grant DE-FG0294ER40823 at Minnesota.

\section{References}

[1] A.D. Linde, Particle Physics And Inflationary Cosmology (Harwood, 1990); K.A. Olive, Phys. Rep. 190 (1990) 181; D. Lyth and A. Riotto, hep-ph/9807278.

[2] A.D. Linde, Phys. Lett. B129 (1983) 181.

[3] K. Enqvist and J. Maalampi, Phys. Lett. B180 (1986) 14.

[4] A.D. Linde, Phys. Lett. B202 (1988) 194.

[5] A.R. Liddle, A. Mazumdar and F.E. Schunck, Phys. Rev. D 58 (1998) 061301.

[6] P. Kanti and K.A. Olive, Phys. Rev. D (1999) in press, hep-ph/9903524.

[7] E.J. Copeland, A. Mazumdar, and N.J. Nunes, astro-ph/9904309.

[8] K.A. Malik and D. Wands, Phys. Rev. D 59 (1999) 123501.

[9] I. Antoniadis, N. Arkani-Hamed, S. Dimopoulos and G. Dvali, Phys. Lett. B436 (1998) 257; N. Kaloper and A. Linde, Phys. Rev. D 59 (1999) 101303; D. Lyth, Phys. Lett. B448 (1999) 191; A. Lukas, B.A. Ovrut and D. Waldram, hep-th/9902071; N. Arkani-Hamed, S. Dimopoulos, N. Kaloper and J. March-Russell, hep-ph/9903224; hep-ph/9903239; J.M. Cline, hep-ph/9904495.

[10] J. Ellis, N. Kaloper, K.A. Olive and J. Yokoyama, Phys. Rev. D 59 (1999) 103503.

[11] A. Chodos and T. Appelquist, Phys. Rev. Lett. 50 (1983) 141.

[12] F. Graziani and K.A. Olive, Phys. Lett. B216 (1989) 31.

[13] T.S. Bunch and P.C.W. Davies, Proc. R. Soc. London A360 (1978) 117; A. Vilenkin and L.H. Ford, Phys. Rev. D 26 (1982) 1231; A.D. Linde, Phys. Lett. B116 (1982) 335; Phys. Lett. B131 (1983) 330; A. Vilenkin, Nucl. Phys. B226 (1983) 527; K. Enqvist, K.W. Ng and K.A. Olive, Nucl. Phys. B303 (1988) 713. 